\title{
Quistes pancreáticos: valor agregado de EUS-FNA sobre EUS y RMI. Experiencia de un hospital universitario de alta complejidad
}

\author{
Inés C Oría, ${ }^{1}$ Juan E Pizzala, ${ }^{1}$ Augusto Villaverde, ${ }^{1}$ Juan Rivera Flores, ${ }^{1}$ Analía Pasqua, \\ Vanina L Pagotto, ${ }^{3}$ Oscar M Mazza, ${ }^{2}$ Víctor Abecia, ${ }^{1}$ Mariano M Marcolongo ${ }^{1}$ \\ ${ }^{1}$ Servicio de Gastroenterología, Hospital Italiano de Buenos Aires. \\ ${ }^{2}$ Servicio de Cirugía General, Hospital Italiano de Buenos Aires. \\ ${ }^{3}$ Departamento de Investigacion, Hospital Italiano de Buenos Aires. \\ Ciudad Autónoma de Buenos Aires, Argentina.
}

Acta Gastroenterol Latinoam 2020;50(2):124-132

Recibido: 26/09/2019 / Aceptado: 13/05/2020 / Publicado online: 29/06/2020

\section{Resumen}

Introducción. La ecoendoscopía (EUS) y la colangiopancreatorresonancia (CPRMN) son herramientas valiosas en la caracterización y el manejo de las lesiones quisticas pancreáticas (LQP). Sin embargo, el diagnóstico preoperatorio de las LQP sigue siendo un desafio. La aspiración con aguja fina (FNA) guiada por EUS nos permite analizar el líquido intraquístico y obtener asi información adicional. Objetivo. Comparar la concordancia entre el diagnóstico de quiste por FNA con la obtenida por dos métodos de diagnóstico por imagen (morfología EUS y CPRM). Evaluar si EUS-FNA ofreció algún beneficio en casos de CPRMN no concluyente. Material y métodos. Se realizó un análisis retrospectivo de los registros informáticos de todas las ecoendoscopias digestivas realizadas entre enero de 2015 y diciembre de 2018 y se incluyó a los pacientes con diagnóstico de quistes pancreáticos. Se analizaron las imágenes, el líquido quistico pancreá-

Correspondencia: Inés C Oría

Perón 4190 (C.P.:1199). Servicio de Gastroenterología, Hospital Italiano de Buenos Aires. Ciudad Autónoma de Buenos Aires, Argentina Tel.: 1540512713

Correo electrónico: ines.oria@hospitalitaliano.org.ar tico (PCF) y el seguimiento. Resultados. De un total de 2238 ecoendoscopías realizadas, 319 presentaron diagnóstico de quiste pancreático. Se realizó punción en 139 pacientes: $62(44,6 \%)$ fueron clasificados como mucinosos según el CEA y/o la citología. La concordancia entre el diagnóstico presuntivo del quiste por punción con el obtenido por EUS fue de 89,2\% (Kappa 0,78, $p<0,001$ ). La concordancia entre el diagnóstico presuntivo del quiste por punción con el obtenido por CPRMN fue de 72,66\% (Kappa 0,41, $p<0,001$ ). Por otro lado, de los 319 pacientes con quistes pancreáticos, 60 (18,8\%) tenian resultado indeterminado en la CPRMN, y por morfología endosonográfica 31 obtuvieron un diagnóstico presuntivo. En cambio, en el grupo de los 139 pacientes punzados, la CPRMN era indeterminada en 40 pacientes $(28,8 \%)$ y la punción diagnosticó 36 de los 40 casos (90\%). Conclusión. En nuestro estudio, la EUS \pm FNA fue superior a la CPRM, especialmente en casos de CPRM no concluyente.

Palabras claves. Ecoendoscopía con punción, quistes pancreáticos, mucinosos.

\section{Pancreatic Cysts: does EUS-FNA adds value over EUS Morphology and MRI. Experience of a Tertiary-Care Academic Medical Center}

\section{Summary}

Background. Endoscopic ultrasound (EUS) and magnetic resonance imaging (MRI) are valuable tools in the 
assessment of cystic pancreatic lesions (CPLs). However, preoperative diagnosis of CPLs still remains a challenge. EUS-guided fine needle aspiration (FNA) provides a method to obtain cyst fluid for analysis to gain additional information. Aim. Compare the concordance between the diagnosis of the cyst by FNA, with that obtained by two diagnostic imaging methods (EUS morphology and MRI). Evaluate if EUS-FNA offered any benefit in cases of inconclusive MRI. Material and methods. We performed a retrospective analysis of a prospectively collected database at a tertiary-care academic medical center between January 2015 and December 2018. All patients who were referred for EUS were reviewed, and patients with diagnosis of pancreatic cysts were included. Imaging, pancreatic cyst fluid (PCF) and follow-up were analyzed. Results. A total of 2238 EUS were performed during the study period and 319 of them had a final diagnosis of pancreatic cyst. FNA was performed on 139 cysts: 62 were diagnosed as mucinous by PCF. The agreement between the diagnosis of the cyst by FNA, with that obtained by EUS morphology was $89.2 \%$ (Kappa 0.78, $p<0.001$ ). The concordance between the diagnosis of the cyst by FNA and that obtained by MRI was 72.66 (Kappa 0.41, $p<0.001$ ). From the 319 patients with pancreatic cysts, 60 (18.8\%) had inconclusive results on MRI and EUS morphology was able to make a diagnosis in 31 of them. When we analyzed the 139 punctured cysts, MRI was indeterminate in 40 patients and FNA diagnosed 36 of the 40 patients (90\%). Discussion. In our study, EUS +/- FNA was superior to $M R I$ specially in cases of inconclusive MRI.

Key words. EUS, EUS-FNA, pancreatic cysts, MRI.

\section{Introducción}

Debido a la evolución y el creciente uso de las técnicas imagenológicas, la detección de los quistes pancreáti$\cos$ ha aumentado en los últimos años. Actualmente, la prevalencia de los quistes incidentales es entre un 2,4 y $13 \%,{ }^{1-3}$ siendo mucho mayor en pacientes con patología pancreática subycente, ${ }^{4-6}$ y llegando hasta el $24 \%$ en un estudio realizado en autopsias. ${ }^{7} \mathrm{Su}$ incidencia aumenta con la edad. ${ }^{1-4}$

Los quistes pancreáticos pueden ser no neoplásicos o neoplásicos, y estos últimos, a su vez, mucinosos y no mucinosos. ${ }^{1-3}$ La diferenciación es crucial, ya que el manejo depende del tipo de quiste.

Muchas veces las lesiones son morfológicamente características y fácilmente distinguibles. Pero, otras veces, a pesar del gran avance de las técnicas diagnósticas, la distinción de las neoplasias quísticas mediante la clínica y la morfología es insatisfactoria. Esto plantea un desafío importante en el manejo de estas lesiones, ya que algunos quistes son lesiones benignas sin potencial neoplásico, pero otras son malignas, o premalignas, como lo son los quistes mucinosos, que son los más frecuentes y precursores de adenocarcinoma pancreático ductal. Debido al dilema diagnóstico planteado por estas lesiones y las posibles consecuencias graves de un diagnóstico incorrecto, muchas veces los pacientes con quistes inofensivos son sobretratados con cirugía, y otras veces pacientes con quistes malignos o premalignos son perdidos. ${ }^{8-10}$

La ecografía endoscópica (EUS) ha revolucionado el diagnóstico de las lesiones quísticas pancreáticas, siendo más precisa que la tomografía computada (TC) y la resonancia magnética (RMN) para diferenciar quistes neoplásicos de no neoplásicos. ${ }^{11,12}$ Provee información de gran certeza sobre las características del quiste, incluyendo la presencia de septos, nódulos, debris y grosor de la pared, y permite además, en forma segura, la punción y el análisis del líquido intraquístico. ${ }^{13-19}$ Actualmente, el test preoperatorio más preciso para diferenciar un quiste mucinoso de uno no mucinoso ha demostrado ser el CEA (antígeno carcinoembrionario) del líquido intraquístico, con una especificidad (E) y sensibilidad (S) de $75 \%$ y $84 \%$ respectivamente, para un valor de corte de $192 \mathrm{ng} / \mathrm{ml}, \mathrm{S}$ del $98 \%$ para un CEA $>800 \mathrm{ng} / \mathrm{ml}$ y E del $94 \%$ para un CEA $<5 \mathrm{ng} / \mathrm{ml} .{ }^{20-23} \mathrm{El}$ objetivo de nuestro estudio fue describir las características de los quistes pancreáticos en los cuales se realizó EUS con y sin punción, y comparar la concordancia entre el diagnóstico presuntivo del quiste por punción con el obtenido por dos métodos diagnósticos de imagen (EUS y RMN). Además, nos propusimos evaluar el valor agregado de la EUS \pm FNA en pacientes con colangiopancreatorresonancia (CPRM) no concluyente.

\section{Material y métodos}

Se realizó un análisis retrospectivo sobre una base de datos electrónica cargada de manera prospectiva, que incluyó a una serie consecutiva de pacientes con quistes pancreáticos que realizaron una ecoendoscopía biliopancreática en el período comprendido entre enero de 2015 y diciembre de 2018, en un hospital privado de la comunidad, centro de referencia en patología pancreática. No hubo un protocolo específico para la realización de EUS; la decisión quedó a cargo del médico tratante. El protocolo fue aprobado por el Comité de Ética institucional. Se recolectaron datos referentes a las características morfológicas de los quistes (número tamaño, localización, presencia de septos, lobulaciones, grosor de la pared, comunicación con conducto pancreático prin- 
cipal, cicatriz central, dilatación del conducto), del líquido intraquístico (color, consistencia, citología, amilasa, CEA), diagnóstico presuntivo según imágenes (EUS y CPRMN) y según punción, tratamiento recibido y datos demográficos (consumo de alcohol y tabaco, antecedentes personales y familiares de enfermedad pancreática). Los quistes se clasificaron como mucinosos o no mucinosos en función de cualquiera de los siguientes parámetros: 1) líquido quístico pancreático (PCF) viscoso, filante (string sign positivo), 2) citología positiva y 3) nivel de CEA $\geq 192 \mathrm{ng} / \mathrm{ml}$. Todos los procedimientos fueron realizados, luego de obtener consentimiento informado del paciente, por endosonografistas entrenados. Se utilizó un procesador Olympus EU-ME1 con ecoendoscopio lineal Olympus (GF-UC140P-AL5) y un procesador Hitachi HI VISION Avius con ecoendoscopio lineal Pentax (EG-3870UTK). Las punciones fueron realizadas con agujas de 25G, 22G y 19G (Cook EchoTip ${ }^{\circledR}$ Ultra ) o 25G, 22G y 19G (Expect Boston Scientific). Para minimizar el riesgo de infección se administró ceftriaxona intravenosa durante el procedimiento, y se indicó ciprofloxacina vía oral los tres días siguientes al procedimiento. Se intentó extraer todo el líquido de los quistes en una única punción. Si el contenido del quiste era suficiente ( $1 \mathrm{ml}$ o más) se colocaba en dos frascos estériles, uno para citología y el otro para fisicoquímico (CEA y amilasa).

Se comparó la concordancia entre el diagnóstico presuntivo del quiste por punción con el obtenido por los dos métodos diagnósticos de imagen (EUS y CPRMN) mediante el estadístico de Kappa. Se expresaron los intervalos de confianza del $95 \%$.

Se consideró un nivel de significación menor al 5\%. El análisis estadístico se realizó con el software Stata 14.

\section{Resultados}

En el período analizado se realizaron 2238 ecoendoscopías. De esas, $319(27,4 \%)$ correspondieron a quistes pancreáticos. El $70 \%$ fueron mujeres, con una edad media de $64,4 \pm 13,5$. Treinta y cinco pacientes $(11 \%)$ presentaban antecedentes de tabaquismo, 15 (4,5\%) de enolismo, y $16(5 \%)$ antecedentes familiares de cáncer de páncreas. El motivo más frecuente por el que se realizó el estudio fue por vigilancia de quistes ya conocidos: 175 (55\%), seguido de hallazgo no concluyente en estudio previo: 102 (32\%), pancreatitis aguda: 20 (6\%), y otros: $22(7 \%)$.

Las características morfológicas de los quistes se resumen en la Tabla 1.

El tipo de lesión más frecuente según la morfología fue similar con los dos métodos, siendo el más frecuente el IPMNII (Gráficos 1 y 2).
Del total de los 319 quistes se punzaron 139 (43,5\%). La punción se realizó a discreción del endoscopista o por pedido del médico tratante.

Las características del líquido intraquístico se resumen en la Tabla 2.

Tabla 1. Características morfológicas de los quistes.

\begin{tabular}{lr}
\hline Características de los quistes & $\mathrm{n}=319$ \\
\hline Numero de quistes: & $198(62 \%)$ \\
1 & $61(19 \%)$ \\
2 & $60(19 \%)$ \\
3 o más & \\
& \\
Tamaño del quiste mayor, media \pm SD & $22,6 \pm 18,5$ \\
Localización del quiste mayor: & \\
Cabeza & $102(32 \%)$ \\
Proceso uncinado & $29(9 \%)$ \\
Cola & $29(9 \%)$ \\
Cuello & $67(21 \%)$ \\
Cuerpo & $92(29 \%)$
\end{tabular}

$\begin{array}{lr}\text { Lobulaciones } & \\ \text { Unilocular } & 223(70 \%) \\ \text { Bilocular } & 19(6 \%) \\ \text { Multilocular } & 77(24 \%)\end{array}$

Septos

No $207(65 \%)$

Sí $112(35 \%)$

Comunicación con conducto principal

Sí

$175(55 \%)$

$144(45 \%)$

Pared del quiste

Fina

$303(95 \%)$

Gruesa

$16(5 \%)$

Contenido del quiste

Líquido

$255(80 \%)$

Mixto

$64(20 \%)$

Cicatriz central

No

297 (93\%)

Sí $22(7 \%)$

Dilatación conducto pancreático

No

$268(84 \%)$

Sí

$51(16 \%)$ 
Gráfico 1. El tipo de quiste más frecuente según la morfología EUS fue el IPMN II.

\section{Diagnóstico presuntivo según morfología EUS}

$60 \%$

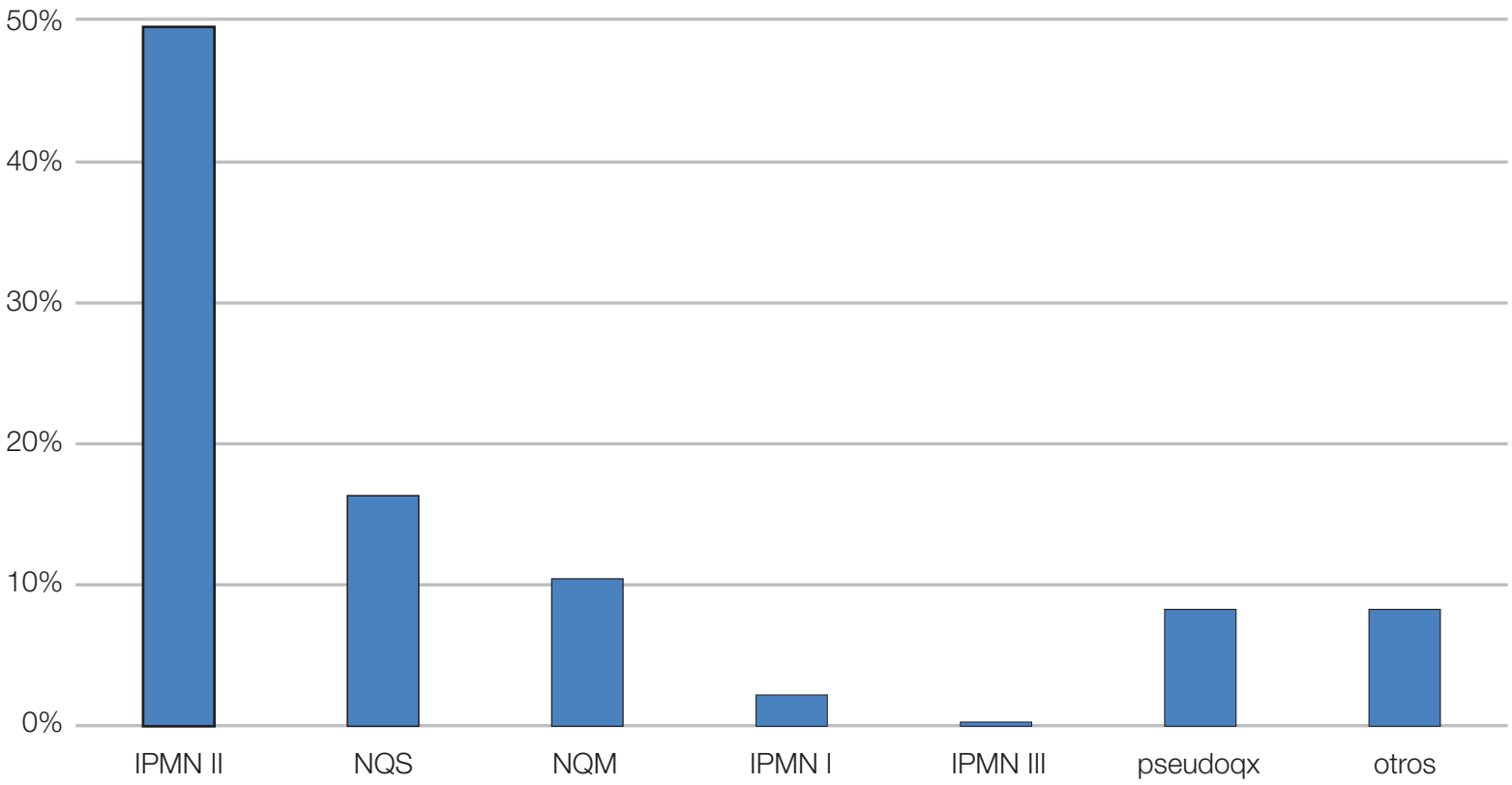

Gráfico 2. El tipo de quiste más frecuente según RMI fue el IPMN II.

\section{Diagnóstico presuntivo según $\mathrm{RMI}$}

$60 \%$

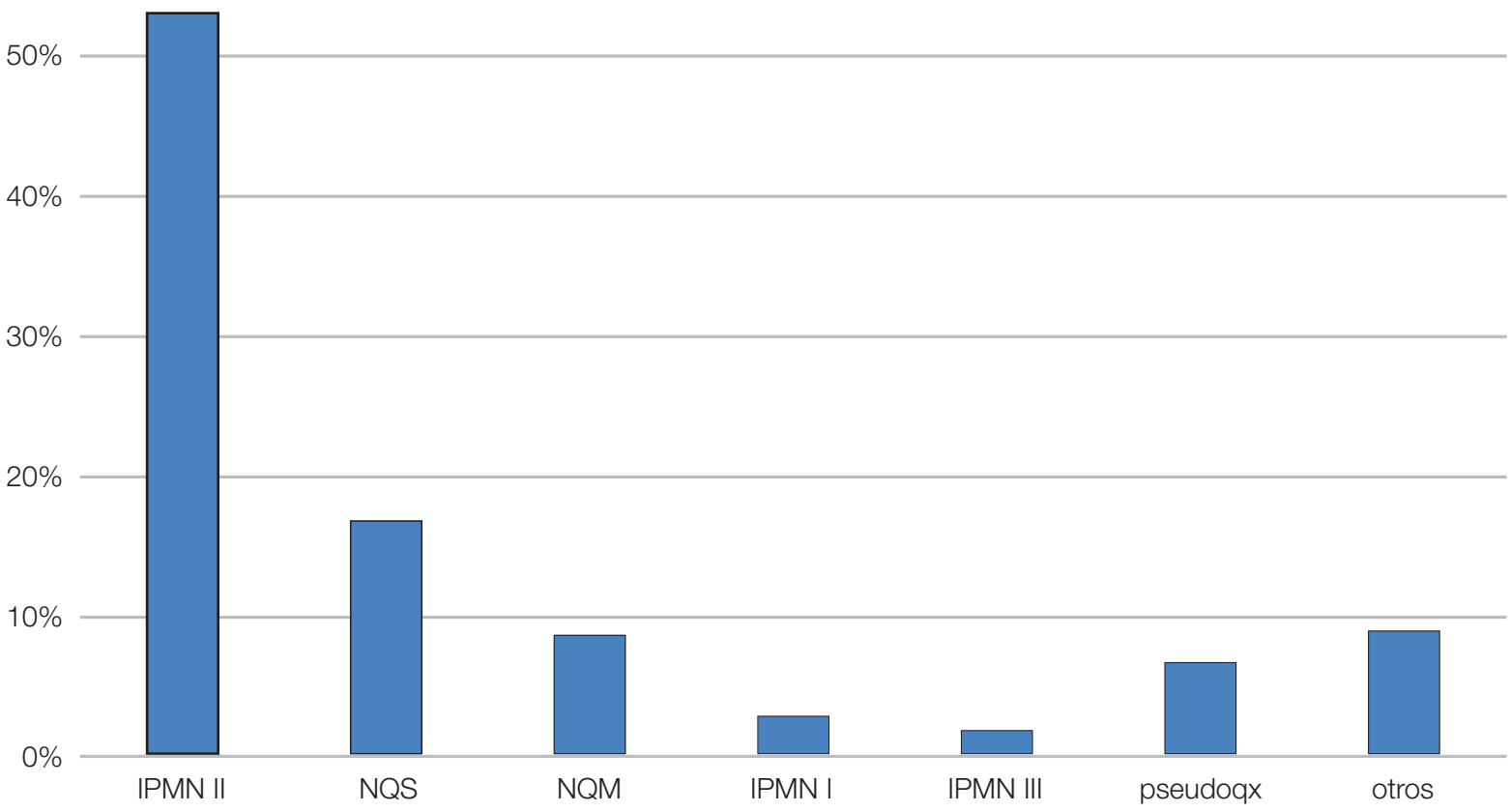


De los 139 quistes punzados, 60 (43,2\%) fueron clasificados como mucinosos según el CEA y/o la citología. La concordancia entre el diagnóstico presuntivo del quiste por punción con el obtenido por EUS fue de 89,2\% (Kappa $0,78, p<0,001)$. La concordancia entre el diagnóstico presuntivo del quiste por punción con el obtenido por CPRMN fue de 72,66\% (Kappa 0,41, $p<0,001$ ).

Por otro lado, de los 319 pacientes con quistes pancreáticos, 60 (18,8\%) tenían resultado indeterminado en la CPRMN, y por morfología endosonográfica 31 pacientes obtuvieron un diagnóstico presuntivo (Gráfico 3). En cambio, en el grupo de los 139 pacientes punzados, la CPRMN era indeterminada en 40 pacientes $(28,8 \%)$ y la punción diagnosticó a 36 de los 40 pacientes (90\%): 13 mucinosos (CEA > $192 \mathrm{ng} / \mathrm{ml}$ y citología + en 4 quistes), 18 serosos (CEA $<5 \mathrm{ng} / \mathrm{ml}$ y citología + en 3 quistes), 4 tumores neuroendocrinos quísticos (TNE) con citología positiva y CEA $<5 \mathrm{ng} / \mathrm{ml}$ en los cuatro pacientes, 1 pseudoquiste (CEA 50 ng/ml, amilasa 25000 ) (Gráfico 4).

No hubo complicaciones intra ni posprocedimiento.

\section{Discusión}

El adenocarcinoma pancreático es una enfermedad devastadora, con una sobrevida menor al 6\% a 5 años. Actualmente es la cuarta causa de muerte por cáncer en
Tabla 2. Características del líquido intraquistico.

Características del líquido quístico

$\mathrm{n}=319$

Color

Amarillento

34

Amarronado

3

Hemático

20

Transparente

Consistencia:

Ligero

Viscoso

\section{Citología}

Diagnóstica

A) Mucinosos

B) No mucinosos

No diagnóstica

\section{CEA}

$>192 \mathrm{ng} / \mathrm{ml}$

< $192 \mathrm{ng} / \mathrm{ml}$

Gráfico 3. De los 319 pacientes con quistes pancreáticos, 60 (18,8\%) tenian resultado indeterminado en la CPRMN (A), y por morfología endosonográfica (B) 31 pacientes obtuvieron un diagnóstico presuntivo.

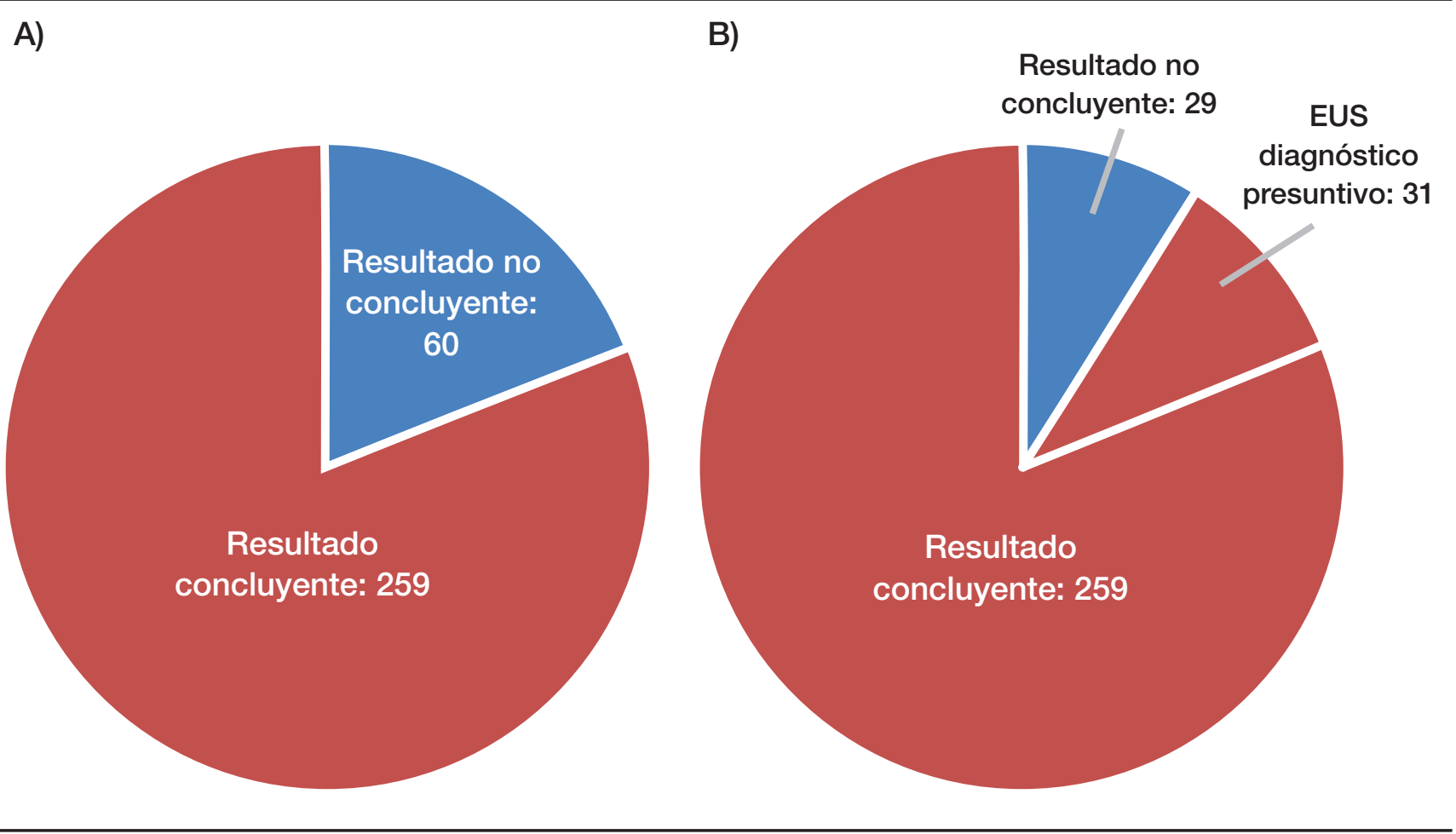


Gráfico 4. En el grupo de los 139 pacientes punzados la CPRMN era indeterminada en 40 pacientes (28,8\%) (A) y la punción diagnosticó a 36 de los 40 pacientes (90\%) (B).

A)

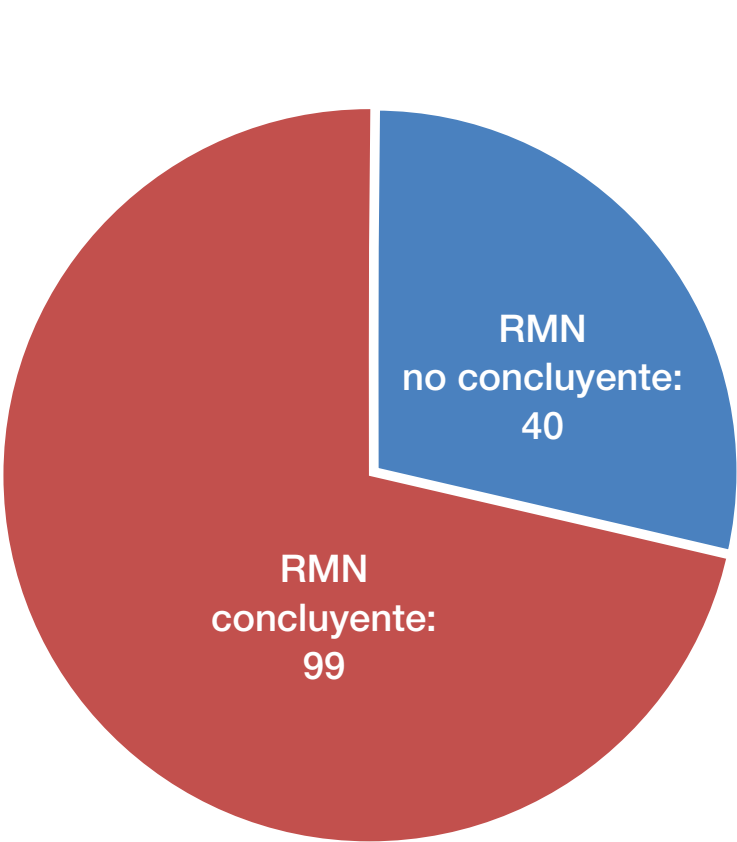

B)

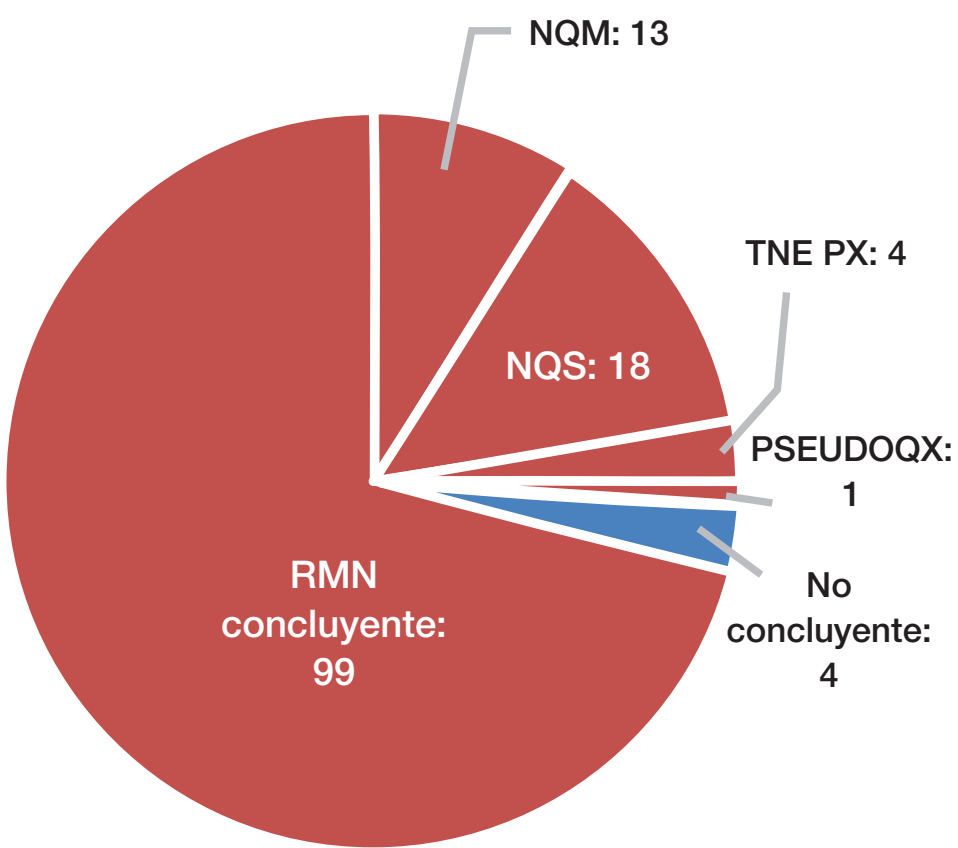

los Estados unidos y se prevé que en 2030 será la segunda. ${ }^{24}$ Dos de sus tres precursores son los quistes mucinosos: IPMN y NQM. Debido a la creciente prevalencia de quistes pancreáticos incidentales, ha aumentado la demanda de un diagnóstico preoperatorio preciso. El manejo de los quistes pancreáticos se basa en la diferenciación de quistes mucinosos de no mucinosos y quistes benignos de malignos. ${ }^{25,26}$ La tomografía computada (el método más comúnmente utilizado) es en general el primer estudio realizado, debido a su gran disponibilidad y su capacidad de detectar quistes. ${ }^{27}$ La RMN es utilizada cada vez con mayor frecuencia para examinar los quistes pancreáticos debido a que las imágenes de CPRMN potenciadas en T2 permiten una evaluación detallada del conducto pancreático principal y de las lesiones quísticas asociadas. ${ }^{28,} 29$ Sin embargo, ambas modalidades de imágenes carecen de precisión en la caracterización de los quistes. ${ }^{30,31}$ Por ejemplo, Fisher y col. ${ }^{31}$ estudiaron la precisión diagnóstica de la TAC en la predicción del potencial maligno de los quistes pancreáticos, y encontraron que el diagnóstico bajo TAC era preciso en un 39\% de los casos, por lo que concluyeron que recomendaban estudios complementarios como la EUS-FNA con análisis del líquido intraquístico para mejorar el diagnóstico. Visser y col. ${ }^{30}$ mostraron la baja precisión diagnóstica de la TAC y RMN en la caracterización de los quistes pancreáticos y mostraron una tasa relativamente alta de diagnósticos incorrectos. Hay algunos trabajos que compararon la precisión diagnóstica de la EUS con la de la TAC y/o RMN. Kim y col..$^{32}$ observaron una precisión diagnóstica similar para EUS y RMN. Kashab y col. ${ }^{11}$ mostraron que la EUS con o sin punción aumentaba el rédito diagnóstico para detectar quistes neoplásicos de 36 a 54\% luego de la TAC/RMN. Du y col..$^{33}$ evaluaron las ventajas de EUS sobre RMN y TC en la evaluación de algunas estructuras específicas de los quistes. Comprobaron que la EUS es superior a TAC y a RMN en la evaluación de estructuras específicas de los quistes pancreáticos, como los nódulos sólidos y septos, y que presenta muchas ventajas sobre las otras dos técnicas de imagen.

En nuestro estudio observamos que EUS con y sin punción fue superior a la CPRMN en el diagnóstico de quistes mucinosos, especialmente cuando la CPRMN fue no concluyente. Al evaluar la concordancia entre el diagnóstico presuntivo por punción y el diagnóstico presuntivo según las imágenes, vimos que entre punción y morfología endosonográfica la concordancia fue buena, mientras que para la CPRMN fue débil. Esta falta de concordancia puede deberse a los pocos pacientes evaluados, aunque hay estudios que demuestran estas observaciones. ${ }^{11,12}$ 
Cuando evaluamos las CPRMN no concluyentes vimos que había 60 de 319 pacientes sin un diagnóstico concluyente, mientras que la mitad de ellas tuvo un diagnóstico presuntivo al realizar EUS. Al evaluar el subgrupo de pacientes punzados, de 139 casos había 40 con CPRMN no concluyente, y de estos, 36 pacientes (90\%) lograron obtener un diagnóstico definitivo con la punción. En estos casos, la EUS + FNA fue fundamental en el diagnóstico de las lesiones quísticas y cambió la conducta en el 90\% de los pacientes con CPRMN inconclusa.

Para definir el diagnóstico de quiste mucinoso utilizamos el CEA, string sign, y la citología. En 2004, un estudio multicéntrico y ciego de Brugge y col. ${ }^{20}$ mostró que, con un valor de corte de $192 \mathrm{ng} / \mathrm{ml}$, los niveles de CEA en el líquido del quiste fueron capaces de diferenciar un MCN de un NMCN con un alto grado de precisión (sensibilidad del 73\%, especificidad del 84\%). Sin embargo, pequeñas series de casos han demostrado que los niveles de CEA pueden no ser tan precisos como se informó anteriormente (sensibilidad del $28-100 \%$, especificidad del $25-100 \%) .{ }^{34,35}$ La citología presenta muy bajo rédito diagnóstico en las lesiones quísticas debido a la baja celularidad del líquido, por lo que su interpretación es muy limitada. ${ }^{36}$ Lo cierto es que hasta el día de hoy no tenemos marcadores preoperatorios $100 \%$ confiables, por lo que es necesario la búsqueda de nuevos marcadores para mejorar la estratificación del riesgo de las neoplasias quísticas pancreáticas. Los marcadores moleculares han mostrado un futuro promisorio en los dos aspectos más importantes en la evaluación de los quistes pancreáticos: el KRAS y GNAS en diferenciación de MCN y NMCN, y TP53, PIK3CA, PTEN, SMAD4, CDKN2A, entre otros, para la evaluación de malignidad en los MCN. ${ }^{37,38}$ Otro marcador descubierto recientemente es la glucosa en el líquido intraquístico. Es un estudio rápido, preciso y de bajo costo. Según nuestro conocimiento, hay solo tres trabajos que observaron que el nivel de glucosa intraquística disminuye significativamente en los quistes mucinosos en comparación con los quistes no mucinosos, presentando una sensibilidad y especificidad de entre $81 \%$ a $95 \%$ y $57 \%$ a $87 \%$, respectivamente. ${ }^{39,40,41}$

Por lo tanto, si bien no contamos con la biopsia como gold standard, utilizamos la combinación del Cea, citología, string sign y seguimiento de al menos seis meses para definir quiste mucinoso o no mucinoso. Otro dato muy importante es que en los NET quísticos, la citología tiene una precisión diagnóstica muy alta, entre 77 y $86 \%$. Además, actualmente, 1 de cada 14 tumores quísticos resecados es un NET quístico, y entre un 10 y $17 \%$ de los NET son quísticos. ${ }^{42}$ Este es otro motivo por el cual es importante siempre realizar citología, por más que normalmente el rédito diagnóstico es muy bajo.

Si bien en este estudio se utilizaron registros electrónicos de pacientes, estos se obtuvieron de la historia clínica electrónica, que es un sistema informático de alta calidad que permite un seguimiento longitudinal y detallado de los pacientes. Otra limitación que presentamos es que muchos de los pacientes que realizaron ecoendoscopía en nuestro centro traían CPRMN de otras instituciones, de diversas calidades, por lo que la comparación entre estos dos métodos no es homogénea.

En los últimos años ha habido una creciente utilización de la ecoendoscopía en nuestro país. Aún son escasos los datos locales de este tipo de lesiones, así como también de la implementación de la ecoendoscopía. Este estudio es importante debido a que es el primero en nuestro país en comparar las diversas técnicas en el diagnóstico diferencial de los quistes pancreáticos. Son necesarios estudios prospectivos para seguir contribuyendo al conocimiento.

Como conclusión, la EUS con punción fue superior a la CPRMN en verificar el diagnóstico de neoplasias mucinosas. La EUS se ha convertido en un procedimiento crucial en el diagnóstico de esta patología, aunque son necesarios nuevos marcadores para mejorar la precisión de este método.

Sostén financiero. Ninguno de los autores ha recibido becas u otro aporte económico para esta investigación.

\section{Referencias}

1. De Jong K, Nio CY, Hermans JJ, Dijkgraaf MG, Gouma DJ, van Eijck CH, Van Heel E, Klass G, Fockens P, Bruno MJ. High prevalence of pancreatic cysts detected by screening magnetic resonance imaging examinations. Clin Gastroenterol Hepatol 2010; 8 (9): 806-811.

2. Laffan TA, Horton KM, Klein AP, Berlanstein B, Siegelman SS, Kawamoto S, Johnson PT, Fishman EK, Hruban RH. Prevalence of unsuspected pancreatic cysts on MDCT. AJR Am J Roentgenol. 2008; 191 (3): 802-807.

3. Lee KS, Sekhar A, Rofsky NM, Pedrosa I. Prevalence of incidental pancreatic cysts in the adult population on MR imaging. Am J Gastroenterol 2010; 105 (9): 2079-2084

4. De Jong K, Buno MJ, Fockens P. Epidemiology, Diagnosis, and Management of Cystic Lesions of the Pancreas. Gastroenterol Res Pract 2012; 2012: 147465.

5. Spinelli KS, Fromwiller TE, Daniel RA, Kiely JM, Nakeeb A, Komorowski RA, Wilson SD, Pitt HA. Cystic pancreatic neoplasms: observe or operate. Ann Surg 2004; 239 (5): 651-657.

6. Zhang XM, Mitchell DG, Dohke M, Holland GA, Parker L. Pancreatic cysts: depiction on single-shot fast spin-echo MR images. Radiology 2002; 223 (2): 547-553.

7. Kimura K, Nagai H, Kuroda A, Muto T, Esaki Y. Analysis of small cystic lesions of the pancreas. Int J Pancreatol 1995; 18 (3): 197-206. 
8. Valsangkar NP, Morales-Oyarvide V, Thayer SP, Ferrone CR, Wargo JA, Warshaw AL, Fernández del Castillo C. 851 resected cystic tumors of the pancreas: a 33-year experience at the Massachusetts General Hospital. Surgery 2012; 152 (Suppl 1): S4-S12.

9. Sahora K, Mino-Kenudson M, Brugge W, Thayer SP, Ferrone CR, Sahani D, Pitman MB, Warshaw AL, Lillemoe KD, Fernández del Castillo CF. Branch duct intraductal papillary mucinous neoplasms: does cyst size change the tip of the scale? A critical analysis of the revised international Consensus Guidelines in a large single-institutional series. Ann Surg 2013; 258: 466-475.

10. Fritz S, Klauss M, Bergmann F, Hackert T, Hartwig W, Strobel O, Bundy BD, Büchler MW, Werner J. Small (Sendai negative) branch-duct IPMNs: not harmless. Ann Surg 2012; 256 (2): 313-320.

11. Khashab MA, Kim K, Lennon AM, Shin EJ, Tignor AS, Amateau SK, Singh VK, Wolfgang CL, Hruban RH, Canto MI. Should We Do EUS/FNA on Patients with Pancreatic Cysts? The Incremental Diagnostic Yield of EUS Over CT/MRI for Prediction of Cystic Neoplasms. Pancreas 2013; 42 (4): 717-721.

12. Ardengh JC, Lopes CV, de Lima-Filho ER, Kemp R, Dos Santos JS. Impact of endoscopic ultrasound-guided fine-needle aspiration on incidental pancreatic cysts. A prospective study. Scandinavian Journal of Gastroenterology 2014; 49: 114-120.

13. Bhutani MS, Gupta V, Guha S, Gheonea DI, Saftoiu A. Pancreatic Cyst Fluid Analysis - A Review. J Gastrointestin Liver Dis 2011; 20 (2): 175-180.

14. Oh HC, Kang H, Brugge W. Cyst Fluid Amylase and CEA Levels in the Differential Diagnosis of Pancreatic Cysts: A Single-Center Experience with Histologically Proven Cysts. Dig Dis Sci 2014; 59 (12): 3111-3116.

15. Brugge W. Diagnosis and management of cystic lesions of the pancreas. J Gastrointest Oncol 2015; 6 (4): 375-388.

16. Lee LS, Clancy T, Kadiyala V, Suleiman S, Conwell DL. Interdisciplinary Management of Cystic Neoplasms of the Pancreas. Gastroenterol Res Pract 2012; 2012: 513163.

17. Thornton GD, McPhail MJ, Nayagam S, Hewitt MJ, Vlavianos $\mathrm{P}$, Monahan KJ. Endoscopic ultrasound guided fine needle aspiration for the diagnosis of pancreatic cystic neoplasms: A metaanalysis. Pancreatology 2013; 13 (1): 48-57.

18. Martin AK, Zhou Z. Endoscopic ultrasound-guided fine-needle aspiration for the diagnosis of pancreatic cysts by combined cytopathology and cystic content analysis. World J Gastrointest Endosc 2015; 7 (15): 1157-1169.

19. Oppong KW, Dawwas MF, Charnley RM, Wadehra V, Elamin K, White S, Nayar M. EUS and EUSeFNA diagnosis of suspected pancreatic cystic neoplasms: Is the sum of the parts greater than the CEA? Pancreatology 2015; 15 (5): 531-537.

20. Brugge W, Lewandrowski K, Lewandrowski E, Centeno B, Szydlo T, Regan S, Fernández del Castillo C, Warshaw AL. Diagnosis of pancreatic cystic neoplasms: a report of the cooperative pancreatic cyst study. Gastroenterology 2004; 126: 1330-1336.

21. Kadiyala V, Lee L. Endosonography in the diagnosis and management of pancreatic cysts. World J Gastrointest Endosc 2015; 7 (3): 213-223.

22. Lewandrowski KB, Southern JF, Pins MR, Compton CC, Warshaw AL. Cyst fluid analysis in the differential diagnosis of pancreatic cysts. A comparison of pseudocysts, serous cystadenomas, mucinous cystic neoplasms, and mucinous cystadenocarcinoma. Ann Surg 1993; 217 (1): 41e7.
23. Van der Waaij LA, Van Dullemen HM, Porte RJ. Cyst fluid analysis in the differential diagnosis of pancreatic cystic lesions: a pooled analysis. Gastrointest Endosc 2005; 62 (3): 383e9

24. Rahib L, Smith BD, Aizenberg R, Rosenzweig AB, Fleshman J, Matrisian L. Projecting Cancer Incidence and Deaths to 2030: The Unexpected Burden of Thyroid, Liver, and Pancreas Cancers in the United States. Cancer Res 2014; 74 (11); 2913-2921.

25. Tanaka M, Chari S, Adsay V, Fernández del Castillo C, Falconi M, Shimizu M, Yamaguchi K, Yamao K, Matsuno S. International consensus guidelines for management of intraductal papillary mucinous neoplasms and mucinous cystic neoplasms of the pancreas. Pancreatology 2006; 6 (12): 17-32.

26. Khalid A, Brugge W. ACG practice guidelines for the diagnosis and management of neoplastic pancreatic cysts. Am J Gastroenterol 2007; 102 (10): 2339-2349.

27. Sahani DV, Kadavigere R, Saokar A, Fernández del Castillo C, Brugge WR, Hahn PF. Cystic pancreatic lesions: a simple imaging-based classification system for guiding management. Radiographics 2005; 25 (6): 1471-1484.

28. Brugge WR. The use of EUS to diagnose cystic neoplasms of the pancreas. Gastrointest Endosc 2009; 69 (2 Suppl): S203-S209.

29. Usuki N, Okabe Y, Miyamoto T. Intraductal mucin-producing tumor of the pancreas: diagnosis by MR cholangiopancreatography. J Comput Assist Tomogr 1998; 22: 875-879.

30. Visser BC, Yeh BM, Qayyum A, Way LW, McCulloch CE, Coakley FV. Characterization of cystic pancreatic masses: relative accuracy of CT and MRI. AJR Am J Roentgenol 2007; 189: 648-656.

31. Fisher WE, Hodges SE, Yagnik V, Morón FE, Wu MF, Hilsenbeck SG, Raijman IL, Brunicardi FC. Accuracy of CT in predicting malignant potential of cystic pancreatic neoplasms. HPB (Oxford) 2008; 10: 483-490.

32. Kim YC, Choi JY, Chung YE, Bang S, Kim MJ, Park MS, Kim KW. Comparison of MRI and endoscopic ultrasound in the characterization of pancreatic cystic lesions. AJR Am J Roentgenol 2010; 195 (4): 947-952.

33. Du C, Chai NL, Linghu EQ, Li HK, Sun LH, Jiang L, Wang XD, Tang P, Yang J. Comparison of endoscopic ultrasound, computed tomography and magnetic resonance imaging in assessment of detailed structures of pancreatic cystic neoplasms. World J Gastroenterol 2017; 23 (17): 3184-3192.

34. Oppong KW, Dawwas MF, Charnley RM, Wadehra V, Elamin K, White S, Nayar M. EUS and EUS/FNA diagnosis of suspected pancreatic cystic neoplasms: Is the sum of the parts greater than the CEA? Pancreatology 2015; 15 (5): 531-537.

35. Gaddam S, Ge PS, Keach JW, Mullady D, Fukami N, Edmundowicz SA, Azar RR, Shah RJ, Murad FM, Kushnir VM, Watson RR, Ghassemi KF, Sedarat A, Komanduri S, Jaiyeola DM, Brauer BC, Yen RD, Amateau SK, Hosford L, Hollander T, Donahue TR, Schulick RD, Edil BH, McCarter M, Gajdos C, Attwell A, Muthusamy VR, Early DS, Wani S. Suboptimal accuracy of carcinoembryonic antigen in differentiation of mucinous and nonmucinous pancreatic cysts: results of a large multicenter study. Gastrointest Endosc 2015; 82 (6): 1060-1069.

36. De Jong K, Poley JW, Van Hooft JE, Visser M, Bruno MJ, Fockens P. Endoscopic ultrasound-guided fine-needle aspiration of pancreatic cystic lesions provides inadequate material for cytology and laboratory analysis: initial results from a prospective study. Endoscopy 2011; 43: 585-590.

37. Theisen BK, Wald AI, Singhi AD. Molecular Diagnostics in the Evaluation of Pancreatic Cysts. Surgical Pathology 2016; 9: 441-456. 
38. Elta GH, Enestvedt BK, Sauer BG, Lennon AM. ACG Clinical Guideline: Diagnosis and Management of Pancreatic Cysts. Am J Gastroenterol 2018; 113: 464-479.

39. Carr RA, Yip-Schneider MT, Simpson RE, Dolejs S, Schneider JG, Wu H, Ceppa EP, Park W, Schmidt CM. Pancreatic cyst fluid glucose: rapid, inexpensive, and accurate diagnosis of mucinous pancreatic cysts. Surgery. 2018; 163 (3): 600-605.

40. Park WG, Wu M, Bowen R, Zheng M, Fitch WL, Pai RK, Wodziak D, Visser BC, Poultsides GA, Norton JA, Banerjee S, Chen AM, Friedland S, Scott BA, Pasricha PJ, Lowe AW, Peltz G. Metabolomic-derived novel cyst fluid biomarkers for pancreatic cysts: glucose and kynurenine. Gastrointest Endosc 2013; 78 (2): 295-302.e2.
41. Zikos T, Pham K, Bowen R, Chen AM, Banerjee S, Friedland S, Dua MM, Norton JA, Poultsides GA, Visser BC, Park WG. Cyst Fluid Glucose is Rapidly Feasible and Accurate in Diagnosing Mucinous Pancreatic Cysts. Am J Gastroenterol 2015; 110 (6): 909-914.

42. Morales-Oyarvide V, Yoon WJ, Ingkakul T, Forcione DG, Casey BW, Brugge WR, Fernández del Castillo C, Pitman MB. Cystic pancreatic neuroendocrine tumors: the value of cytology in preoperative diagnosis. Cancer Cytopathol 2014; 122 (6): 435-444. 